\title{
Maximal nonnegative perturbation of a nonnegative matrix
}

\author{
B. Haut (Aspirant FNRS), G. Bastin, and P. Van Dooren
}

UCL-CESAME**

Avenue G. Lemaitre, 4

1348 Louvain-la-Neuve, Belgium

\{haut, bastin, vdooren\}@inma.ucl.ac.be

Summary. For a class of positive matrices $A+K$ with a stable positive nominal part $A$ and a structured positive perturbation part $K$, we address the problem of finding the largest admissible perturbation such that the global matrix remains stable. Theoretical bounds on the size of this set are derived and an algorithm for constructing a set of admissible perturbation is presented.

Key words: Nonnegative matrices, stability, compartiemental systems

\section{Introduction}

A linear time-invariant discrete-time system

$$
x(k+1)=A x(k)
$$

is known to be stable if and only if $\rho(A)<1$.

Models of real world dynamical phenomena often involve positive quantities. A dynamical system (1) is called positive if any trajectory of the system starting in the positive orthant $\mathbb{R}_{+}^{n}$ remains in $\mathbb{R}_{+}^{n}$. In this case, the matrix $A$ has only real positive entries. In many cases, it may be useful to consider systems with a known "nominal" part $A$ and a unknown part $K$ which may represent uncertainty :

$$
x(k+1)=(A+K) x(k) .
$$

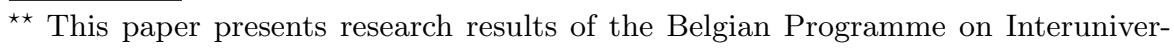
sity Attraction Poles, initiated by the Belgian Federal Science Policy Office. The scientific responsibility rests with its author(s). 
The robustness of (2) will then depend on the size of the set $S$ such that

$$
\rho(A+K)<1 \quad \forall K \in S .
$$

One particular approach consists of considering structured matrices $K=$ $E_{1} \Delta E_{2}^{T}$ where $\Delta$ is the unknown disturbance and $E_{1}$ and $E_{2}$ are fixed matrices. The problem is then to find the stability radius of $A$ with respect to nonnegative perturbations of structure $\left(E_{1}, E_{2}^{T}\right)$ which is defined by

$$
r_{\mathbb{R}_{+}}\left(A ; E_{1}, E_{2}^{T}\right)=\inf \left\{\|\Delta\| ; \Delta \geq 0, \rho\left(A+E_{1} \Delta E_{2}^{T}\right) \geq 1\right\} .
$$

All perturbations in the following set $S=\left\{E_{1} \Delta E_{2}^{T} \mid\|\Delta\|<r_{\mathbb{R}_{+}}\left(A ; E_{1}, E_{2}^{T}\right)\right\}$ are then shown to yield a stable system $A+K$. This problem is solved in [3] and a computable formula is provided.

In this paper we extend these results into a particular direction. We will only consider perturbations matrices $\Delta$ in the set $\mathcal{D}$ of nonnegative diagonal matrices $\mathcal{D}=\left\{\operatorname{diag}\left\{k_{1}, \ldots, k_{m}\right\} \mid k_{i} \geq 0\right\}$. The parameters $k_{i}$ are the socalled free parameters occurring in the matrix $K, E_{1}$ and $E_{2}$ are two matrices placing the elements in appropriate positions in $K$. The two matrices $E_{1}$ and $E_{2}^{T}$ have the following properties : there is a non-zero element in row $i$ and column $j$ of $E_{1}$ if $k_{j}$ is present in row $i$ of $K$ and of $E_{2}^{T}$ if $k_{i}$ is present in column $j$ of $K$. We clarify this by an example : if

$$
K=\left(\begin{array}{ccc}
2 k_{1} & 0 & 0 \\
0 & 0 & k_{2} \\
k_{1} & 0 & 0
\end{array}\right)
$$

then

$$
\Delta=\left(\begin{array}{cc}
k_{1} & 0 \\
0 & k_{2}
\end{array}\right), \quad E_{1}=\left(\begin{array}{ll}
2 & 0 \\
0 & 1 \\
1 & 0
\end{array}\right), \quad E_{2}^{T}=\left(\begin{array}{lll}
1 & 0 & 0 \\
0 & 0 & 1
\end{array}\right) .
$$

We will restrict ourselves to matrices $K$ for which both $E_{1}$ and $E_{2}$ are nonnegative as well : $E_{1} \geq 0, E_{2} \geq 0$.

The problem is to find the biggest set $S_{\mathcal{D}} \subseteq\left\{E_{1} \Delta E_{2}^{T} \mid \Delta \in \mathcal{D}\right\}$ containing the origin such that

$$
\rho(A+K)<1 \quad \forall K \in S_{\mathcal{D}}
$$

where $A, E_{1}, E_{2}, \Delta$ are nonnegative matrices. Let us point out that this is in fact a starlike set. A starlike set is a set containing the origin and all elements between the origin and any other element of the set. Since for $0<\alpha<1$ we have $A+\alpha K \leq A+K$, then $\rho(A+\alpha K) \leq \rho(A+K)<1$. This implies that for every $K \in S_{\mathcal{D}}$ we also have that all $\alpha K$ between the origin and $K$ belong to $S_{\mathcal{D}}$. 
On the one side, the problem solved in [3] is more general because it does not assume that the perturbation $\Delta$ is diagonal. But, on the other side, when $\Delta$ is diagonal, their problem is more restrictive than the one addressed in this paper. All the operator norms induced by an arbitrary monotonic norm on $\mathbb{R}^{n}$ of a diagonal matrix are equal to the maximum of the elements of the matrix. It means that the only considered sets $S$ in [3] are those with $k_{i} \leq k_{i}^{m}$ and $k_{1}^{m}=\ldots=k_{n}^{m}$. If these constraints are not assumed, it is possible to find a set $S_{\mathcal{D}}$ that is bigger than $S$. We will show that for such type of perturbations there exists a maximum starlike set $S_{\mathcal{D}}=\left\{E_{1} \Delta E_{2}^{T} \mid \Delta \in \mathcal{D}\right\}$ for which all matrices $K$ in $S_{\mathcal{D}}$ are stable and we will describe the boundary of this set.

\section{Maximal perturbation of nonnegative matrices}

First we develop some new theoretical results and we then present the corresponding algorithm.

\subsection{Theoretical results}

This section is structured as follows :

- We show that, in certain cases, the problem may be decoupled in smaller subproblems.

- If the size of each subproblem is small enough, we may obtain an analytical expression for a necessary and sufficient condition.

- If the size of the subproblem is too high, it is possible to obtain a necessary condition similar to the one of [3] in terms of the maximum of the $k_{i}$ involved in the subproblem.

Since $K=E_{1} \Delta E_{2}^{T}$ is nonnegative and since the eigenvalues are continuous functions of the matrix elements, we have that the critical switch between $\rho<1$ and $\rho \geq 1$ will occur when

$$
\rho\left(A+E_{1} \Delta E_{2}^{T}\right)=1 \quad \text { (continuity). }
$$

Working only with positive matrices, we have that the spectral radius is also an eigenvalue and hence the above condition is equivalent to

$$
\operatorname{det}\left(A+E_{1} \Delta E_{2}^{T}-I\right)=0
$$

and

$$
\operatorname{det}\left(E_{1} \Delta E_{2}^{T}-(I-A)\right)=0 .
$$

Since $\operatorname{det}(I-A) \neq 0(\rho(A)<1)$ we can multiply the previous equation by $\operatorname{det}(I-A)^{-1}$ to obtain 


$$
\operatorname{det}\left((I-A)^{-1} E_{1} \Delta E_{2}^{T}-I\right)=0 .
$$

Using the fact that $\operatorname{det}(M N-I)=0 \Leftrightarrow \operatorname{det}(N M-I)=0$, this is also equivalent to

$$
\operatorname{det}\left(E_{2}^{T}(I-A)^{-1} E_{1} \Delta-I\right)=0
$$

where $M \triangleq E_{2}^{T}(I-A)^{-1} E_{1}$ is nonnegative since $(I-A)^{-1}=\sum_{i=1}^{\infty} A^{i}$ and $E_{1}, E_{2}$ are nonnegative. We can use Lemma 2.1 (see [1]) to put $M$ under a normal form $\hat{M}$.

Lemma 2.1 Every nonnegative matrix $A$ has a normal form which can obtained under congruent permutation:

$$
\hat{A}=P A P^{T}=\left(\begin{array}{ccc}
\hat{A}_{11} & & 0 \\
& \ddots & \\
& & \hat{A}_{m m}
\end{array}\right)
$$

where each diagonal block $\hat{A}_{i i}$ is square, irreducible or just a $1 \times 1$ zero bock.

Applying the same permutation to $\Delta$, we define $\hat{\Delta}=P \Delta P^{T}$ and have

$$
\operatorname{det}(\hat{M} \hat{\Delta}-I)=0 .
$$

This clearly decomposes in a number of decoupled problems

$$
\operatorname{det}\left(\hat{M}_{i i} \hat{\Delta}_{i i}-I\right)=0 \text {. }
$$

Let us solve the subproblems :

- $\hat{M}_{i i}=0$ then $\operatorname{det}\left(\hat{M}_{i i} \hat{\Delta}_{i i}-I\right) \neq 0$ for all bounded $\hat{\Delta}_{i i}$;

- $\hat{M}_{i i} \neq 0$ and irreducible. If the size $n_{i}$ of $\hat{M}_{i i}=\left[a_{r, c}\right]_{r, c=1}^{n_{i}}$ is small enough, the problem can be exactly solved.

- If $n_{i}=1$, the solution is trivial $\begin{cases}\operatorname{det}\left(a_{11} k_{1}-1\right) \neq 0 & \text { for } k_{1}<a_{11}^{-1} \\ \operatorname{det}\left(a_{11} k_{1}-1\right)=0 & \text { for } k_{1}=a_{11}^{-1}\end{cases}$

- If $n_{i}=2$, we have $\operatorname{det}\left(\left(\begin{array}{ll}a_{11} & a_{12} \\ a_{21} & a_{22}\end{array}\right)\left(\begin{array}{cc}k_{1} & 0 \\ 0 & k_{2}\end{array}\right)-I\right)=0$ or equivalently :

$$
\left(a_{11} a_{22}-a_{12} a_{21}\right) k_{1} k_{2}-a_{11} k_{1}-a_{22} k_{2}+1=0 .
$$

The stable region for the $k_{1}, k_{2}$ is thus a starlike set whose boundary is defined by (5) and $k_{1,2}=0$ (see Fig. 1).

- If $n_{i}=3$, we have $\operatorname{det}\left(\hat{M}_{i i} \hat{\Delta}_{i i}-I\right)=0$ when

$$
\begin{gathered}
\operatorname{det}\left(\hat{M}_{i i}\right) k_{1} k_{2} k_{3}+\left(a_{21} a_{12}-a_{11} a_{22}\right) k_{1} k_{2}+\left(a_{13} a_{31}-a_{11} a_{33}\right) k_{1} k_{3} \\
+\left(a_{23} a_{32}-a_{22} a_{33}\right) k_{2} k_{3}+a_{11} k_{1}+a_{22} k_{2}+a_{33} k_{3}-1=0 .
\end{gathered}
$$

The stable region for the $k_{i}$ is thus also a starlike set whose boundary is defined by (6) and $k_{1,2,3}=0$ (see Fig. 2). 


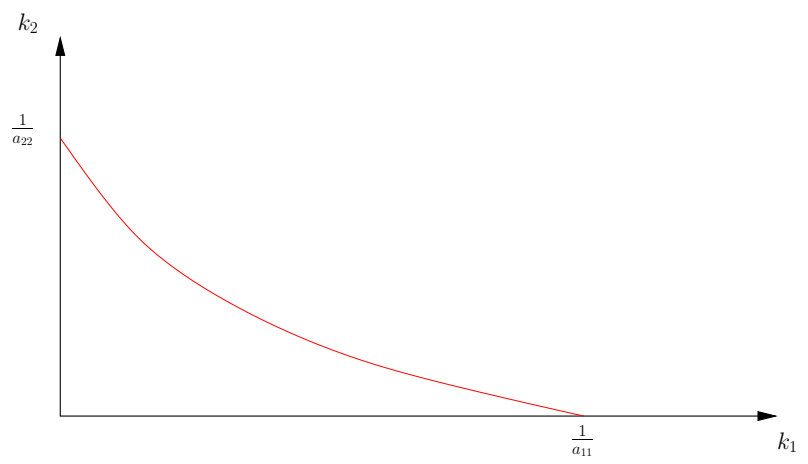

Fig. 1. The largest set of the parameter $k_{1}$ and $k_{2}$ containing the origin such that $A+E_{1} \Delta E_{2}^{T}$ is stable.

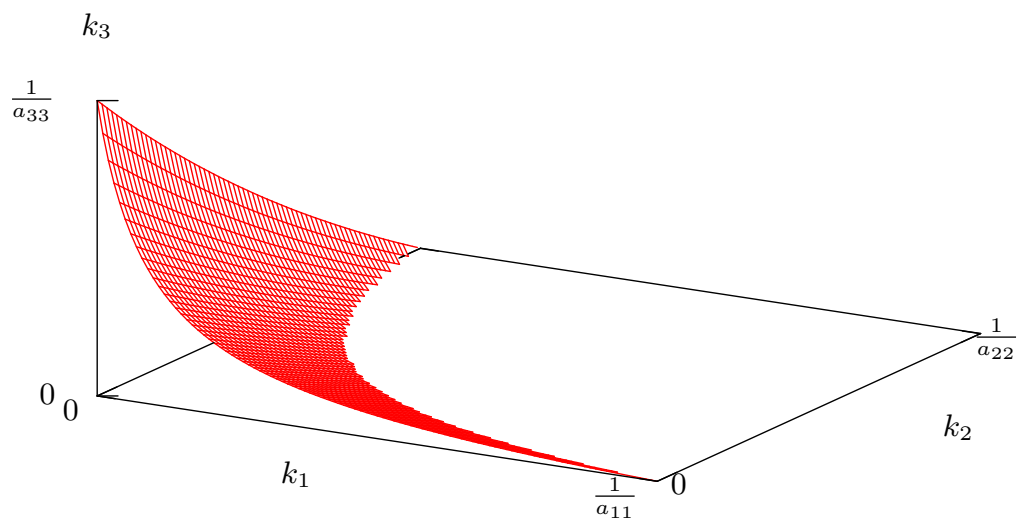

Fig. 2. The boundary of the largest set $\left(k_{1}, k_{1}, k_{3}\right)$ containing the origin such that $A+E_{1} \Delta E_{2}^{T}$ is stable

- It may happen that a coefficient $k_{i}$ appears in different blocks $\hat{\Delta}_{i i}$. For example, if

$$
A=\left(\begin{array}{llll}
a_{11} & a_{12} & & \\
a_{21} & a_{22} & & \\
& & a_{33} & a_{34} \\
& & a_{43} & a_{44}
\end{array}\right), \quad K=\left(\begin{array}{llll}
k_{1} & & & \\
& k_{2} & & \\
& & k_{3} & \\
& & & k_{1}
\end{array}\right)
$$

then 


$$
\hat{M}_{11}=\left(\begin{array}{ll}
k_{1} & \\
& k_{2}
\end{array}\right), \quad \hat{M}_{22}=\left(\begin{array}{ll}
k_{3} & \\
& k_{1}
\end{array}\right) .
$$

In this case, the admissible set for $\left(k_{1}, k_{2}, k_{3}\right)$ is simply the intersection of the two sets obtained by analysing the two subproblems. This is illustrated in Figure 3.

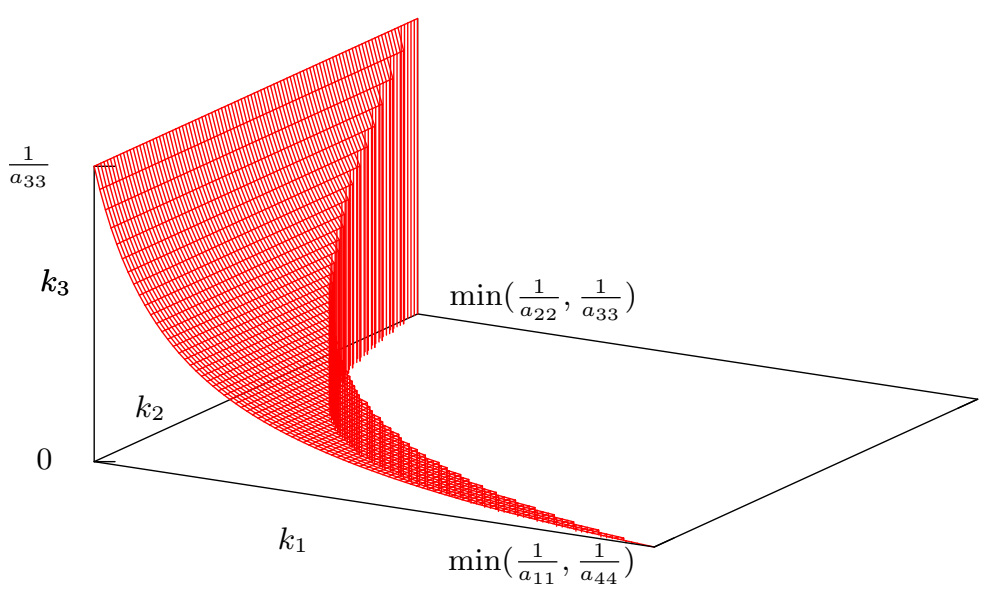

Fig. 3. The admissible set is the intersection of the two admissible sets.

Lemma 2.2 An irreducible matrix $M$ of spectral radius $\rho$ with a nonzero spectral radius has a unique positive Perron vector $x$

$$
M x=\rho x \quad x>0, \rho>0 .
$$

- If the size $n_{i}$ is too large, it may be easier to find a necessary condition function of the maximum of the $k_{i}$. Let $\rho_{i}$ be the spectral radius of $M_{i i}$ then

$$
\begin{array}{ll}
\operatorname{det}\left(\hat{M}_{i i} \hat{\Delta}_{i i}-I\right) \neq 0 & \text { for } \hat{\Delta}_{i i}<\rho_{i}^{-1} I \\
\operatorname{det}\left(\hat{M}_{i i} \hat{\Delta}_{i i}-I\right)=0 & \text { for } \hat{\Delta}_{i i}=\rho_{i}^{-1} I
\end{array}
$$

Proof. Let $x_{i}$ be the Perron vector of the irreducible matrix $\hat{M}_{i i}$. Following Lemma 2.2 (see [1]), we have

$$
\hat{M}_{i i} x_{i}=\rho_{i} x_{i}, x_{i}>0
$$


then clearly

$$
\left(\hat{M}_{i i} \rho_{i}^{-1} I-I\right) x_{i}=0 \quad \hat{\Delta}_{i i}=\rho_{i}^{-1} I
$$

Also for $\hat{\Delta}_{i i}<\rho_{i}^{-1} I$

$$
\operatorname{det}\left(\hat{M}_{i i} \hat{\Delta}_{i i}-I\right) \neq 0
$$

since there exists a scaling

$$
\left\|D^{-1} \hat{M}_{i i} D\right\|_{\infty}=\rho_{i}
$$

and clearly

$$
\left\|D^{-1} \hat{M}_{i i} \hat{\Delta}_{i i} D\right\|_{\infty}=\left\|D^{-1} \hat{M}_{i i} D \hat{\Delta}_{i i}\right\|_{\infty}<1
$$

The problem may thus be split into several subproblems. If the subproblems are small enough, we may have some analytical necessary and sufficient conditions. If the subproblems are more complex, to ensure that $\rho\left(A+E_{1} \Delta E_{2}^{T}\right)<1$, we may impose for each $\hat{M}_{i i} \neq 0, \hat{\Delta}_{i i}<\rho_{i}^{-1} I$ and for each $\hat{M}_{i i}=0$ no bound on $\left\|\hat{\Delta}_{i i}\right\|$.

Therefore we can claim that all matrices $\Delta$ in the following set

$S=\left\{\Delta \mid\left(\begin{array}{lll}\hat{\Delta}_{11} & & \\ & \ddots & \\ & & \hat{\Delta}_{m m}\end{array}\right\}=P \Delta P^{T} \quad \hat{\Delta}_{i i}<\left\{\begin{array}{ll}\text { any bounded value } & \text { if } \rho_{i}=0 \\ \rho_{i}^{-1} I & \text { if } \rho_{i} \neq 0\end{array}\right\}\right.$

are such that (3) holds.

\subsection{Algorithm}

The results presented in the previous section can be used to construct the set $S$. This set can be constructed as follow :

1. Compute the matrix $M \triangleq E_{2}^{T}(I-A)^{-1} E_{1}$ and perform permutations to put it under the normal form (4). This can be done by applying the following algorithm :

a) Use Tarjan's algorithm [4] to find the set of strongly connected subgraphs associated to the graph $G$ defined by the Adjacency Matrix $M^{a d}\left(M_{i, j}^{a d}=1\right.$ if $M_{i, j} \neq 0, M_{i, j}^{a d}=0$ otherwise $)$.

b) Consider a new graph $G^{\prime}$ whose nodes represent the strongly connected subgraphs : two nodes $i$ and $j$ of $G^{\prime}$ are connected if there exists one edge between a node of $G$ in the subgraph $i$ and a node of $G$ in the subgraph $j$. The adjacency matrix of this new graph $G^{\prime}$ can be computed simply from $M^{a d}$ by first summing up the rows corresponding to the same subgraph and then summing up the columns corresponding to the same subgraph. 
c) Identify a leaf $i$ of the graph $G^{\prime}$ (which always exists because there is no cycle in $G^{\prime}$ ) and permute the columns and the rows of $M$ corresponding to the subgraph $i$ at the beginning of the matrix. Suppress node $i$ from $G^{\prime}$. Repeat 1c until $M$ is in canonical form (4).

2. Compute the spectral radius $\rho_{i}$ of each block $\hat{M}_{i i}$ on the diagonal of $M$.

3. Apply to the vector

$$
v=(\underbrace{\rho_{1}^{-1}, \ldots, \rho_{1}^{-1}}_{\operatorname{size}\left(M_{11}\right)}, \rho_{2}^{-1}, \ldots, \rho_{m-1}^{-1}, \underbrace{\rho_{m}^{-1}, \ldots, \rho_{m}^{-1}}_{\operatorname{size}\left(M_{m m}\right)})
$$

the permutation inverse to the one apply to $M$ to obtain the vector $v^{\prime}$.

It can be now claimed that, if $\left(k_{1}, \ldots, k_{n}\right) \in\left\{\left[0, v_{1}^{\prime}\right], \ldots\left[0, v_{n}^{\prime}\right]\right\}$ then

$$
\rho\left(A+E_{1}\left(\begin{array}{ccc}
k_{1} & & \\
& \ddots & \\
& & k_{n}
\end{array}\right) E_{2}^{T}\right)<1
$$

\section{Conclusions}

In this paper, we have considered the problem of finding the largest perturbation such that a positive matrix remains stable. We have extended the results of [3] in a particular case where the problem can be decoupled in smaller subproblems, allowing to increase some parameter values and to get necessary and sufficient analytical conditions. In an extended version of this paper [2], an application of this approach to the stability analysis of networks of conservation laws is presented in details. Some illustrations of this application will also be shown at the conference.

\section{References}

1. F. R. Gantmacher. The Theory of Matrices. New York, NY, USA, 1959.

2. B. Haut, G. Bastin, and P. Van Dooren. Maximal nonnegative perturbation of a nonnegative matrix : theoretical bounds and application to the stability analysis of systems of conservation laws. submitted for publication.

3. D. Hinrichsen and N. K. Son. Stability radii of positive discrete-time systems under affine parameter perturbations. International J. Robust and Nonlinear Control, 8:1169-1188, 1998.

4. R. Tarjan. Depth first search and linear graph algorithms. SIAM Journal of Computing, 1(2):140-160, june 1972. 